\title{
Ocorrência e perfil de sensibilidade e resistência de bactérias isoladas de culturas de aspirados traqueais de um Hospital Universitário do Sertão de Pernambuco
}

\author{
Occurrence and profile of sensitivity and resistance of bacteria isolated from tracheal aspirates \\ cultures of a University Hospital of the Sertão of Pernambuco \\ Ocurrencia y perfil de sensibilidad y resistencia de bacterias aisladas de cultivos de aspirados \\ traqueales de un Hospital Universitario del Sertão de Pernambuco
}

\author{
Gabriela Ramos Gonçalves \\ ORCID: https://orcid.org/0000-0002-1258-2074 \\ Universidade Federal do Vale do São Francisco, Brasil \\ E-mail: ramosgoncalves.gabriela@gmail.com \\ Ricardo Santana de Lima \\ ORCID: https://orcid.org/0000-0002-1279-3445 \\ Universidade Federal do Vale do São Francisco, Brasil \\ E-mail: ricardo.lima@univasf.edu.br \\ Kátia Suely Batista Silva \\ ORCID: https://orcid.org/0000-0001-6146-4229 \\ Universidade Federal do Vale do São Francisco, Brasil \\ E-mail: katia.regina@ebserh.gov.br \\ Katia Regina de Oliveira \\ ORCID: https://orcid.org/0000-0002-3049-0827 \\ Universidade Federal do Vale do São Francisco, Brasil \\ E-mail: katia.regina@ebserh.gov.br \\ Marcos Duarte Guimarães \\ ORCID: https://orcid.org/0000-0002-9458-5649 \\ Universidade Federal do Vale do São Francisco, Brasil \\ E-mail: marcos.guimaraes@ebserh.gov.br \\ Carine Rosa Naue \\ ORCID: https://orcid.org/0000-0003-4215-3606 \\ Universidade Federal do Vale do São Francisco, Brasil \\ E-mail: carine.naue@ebserh.gov.br
}

\begin{abstract}
Resumo
As infecções hospitalares são um desafio para a saúde pública, pois impactam no prognóstico do paciente e nos custos hospitalares. O objetivo deste estudo foi avaliar a ocorrência e o perfil bacteriano de aspirados traqueais de pacientes internados na Unidade de Terapia Intensiva. Trata-se de um estudo retrospectivo, descritivo e documental, tendo como fonte as planilhas disponibilizadas pelo laboratório de Análises Clínicas do Hospital Universitário. Dos 307 laudos analisados em 2017, 142 estavam positivos, e em 2018 dentre os 319, 213 foram positivos. O micro-organismo de maior ocorrência nos dois anos foi Acinetobacter baumannii, seguido de Pseudomonas aeruginosa, Staphylococcus aureus e Klebsiella pneumoniae. Acinetobacter baumannii apresentou resistência à diversos betalactâmicos, dentre eles o meropenem e o imipenem, e para a ampicilina + sulbactam; sendo sensível a colistina e a tigeciclina. Os isolados de $P$. aeruginosa de 2017 e 2018 foram resistentes a: meropenem, imipenem, ceftazidima, cefepima, ciprofloxacino e levofloxacino; no entanto, para a piperacilina + tazobactam observou-se resistência nas amostras obtidas em 2018. S. aureus foi resistente a clindamicina, ao sulfametoxazol + trimetoprima e a oxacilina; apresentou sensibilidade a linezolida, tigecliclina e vancomicina. Os isolados de K. pneumoniae demonstraram resistência, dentre outras drogas, ao imipenem, meropenem, piperacilina + tazobactam; por outro lado, foram sensíveis a amicacina e a colistina. As resistências observadas denotam a importância do uso racional dos antimicrobianos e fornecem dados para a criação de protocolos de antibioticoterapia empírica para o tratamento dos pacientes internados na Instituição.
\end{abstract}

Palavras-chave: Bactérias; Infecção; Unidades de Terapia Intensiva; Hospital.

\section{Abstract}

Hospital infections are a challenge for public health, given their impact on patient prognosis and hospital costs. The objective of this article was to evaluate the bacterial occurrence and the bacterial profile of tracheal aspirates of patients admitted to the ICU. Retrospective, descriptive and documentary study, having as source spreadsheets available by the Laboratory of Clinical Analysis of the University Hospital. Results Of the 307 reports analyzed in 2017, 142 were positive, and in 2018 out of 319, 213 were positive. The most frequent microorganism in the two years was 
Acinetobacter baumannii, followed by Pseudomonas aeruginosa, Staphylococcus aureus and Klebsiella pneumoniae. Acinetobacter baumannii showed resistance to several bethalactamics, including meropenem and imipenem and ampicillin sulbactam; sensitive to colistin and tigecycline. The isolates of $P$. aeruginosa from 2017 and 2018 were resistant to: meropenem, imipenem, ceftazidima, cefepima, ciprofloxacin and levofloxacin. However, for piperacillin tazobactam resistance was observed in the samples obtained in 2018. S. aureus was resistant to clindamycin, sulfamethoxazole trimethoprima and oxacillin; showed sensitivity to linezolid, tigeclicline and vancomycin. $K$. pneumoniae isolates showed resistance, among other drugs, to imipenem, meropenem, piperacillin tazobactam; on the other hand, amicacin and colistin were sensitive. The observed resistances denote the importance of the rational use of antimicrobials and provide data for the creation of empirical antibiotic therapy protocols for the treatment of patients hospitalized in the Institution.

Keywords: Bacteria; Infection; Intensive Care Units; Hospitals.

\section{Resumen}

Las infecciones hospitalarias son un desafío para la salud pública, dado su impacto en el pronóstico del paciente y los costos hospitalarios. El objetivo de este artículo fue evaluar la ocurrencia bacteriana y el perfil bacteriano de los aspirados traqueales de pacientes ingresados en la UCI. Estudio retrospectivo, descriptivo y documental, teniendo como fuente hojas de cálculo disponibles por el laboratorio de Análisis Clínicos del Hospital Universitario. De los 307 informes analizados en 2017, 142 fueron positivos y en 2018 de 319, 213 fueron positivos. El microorganismo más frecuente en los dos años fue Acinetobacter baumannii, seguido de Pseudomonas aeruginosa, Staphylococcus aureus y Klebsiella pneumoniae. Acinetobacter baumannii mostró resistencia a varios bethalactamics, incluyendo meropenem e imipenem y ampicilina sulbactam; sensibles a la colistina y la tigeciclina.Los aislados de P. aeruginosa de 2017 y 2018 fueron resistentes a: meropenem, imipenem, ceftazidima, cefepima, ciprofloxacino y levofloxacino. Sin embargo, para la resistencia a la piperacillin tazobactam se observó en las muestras obtenidas en 2018. S. aureus era resistente a la clindamicina, sulfamethoxazole trimethoprima y oxacillina; mostró sensibilidad a la linezolid, tigeclina y vancomicina. Los aislados $K$. pneumoniae mostraron resistencia, entre otros fármacos, a imipenem, meropenem, piperacillin tazobactam; por otro lado, la amicacina y la colistina eran sensibles. Las resistencias observadas denotan la importancia del uso racional de los antimicrobianos y proporcionan datos para la creación de protocolos empíricos de terapia antibiótica para el tratamiento de pacientes hospitalizados en la Institución.

Palabras Clave: Bacterias; Infección; Unidades de Cuidados Intensivos; Hospitales.

\section{Introdução}

A infecção hospitalar (IH) é definida como as infecções que são adquiridas após 48 a 72 horas da admissão no serviço hospitalar e que ocorrem durante o período de internação ou após a alta, desde que esteja relacionada com o tempo de internação ou ainda aos procedimentos realizados no âmbito hospitalar (Babady, 2016; Cardoso et al., 2014; World Health Organization, 2016). Infecções adquiridas na comunidade, em pacientes que com frequência são expostos a procedimentos invasivos em locais que fornecem cuidados em saúde, foram classificadas como infecções associadas a cuidados de saúde (IACS), uma vez que são semelhantes às infecções hospitalares (Calik Basaran \& Ascioglu, 2017). As IACS são também responsáveis por alta taxa de morbidade e mortalidade e elevam os custos da saúde (Al-Tawfiq \& Tambyah, 2014). Em países subdesenvolvidos elas são uma importante causa de mortalidade, superando casos de câncer de mama e de próstata e acidentes de trânsito (Alp \& Damani, 2015).

Os fatores de risco para adquirir uma infecção hospitalar incluem um longo tempo de permanência hospitalar, comprometimento imune dos pacientes, realização de cirurgias invasivas, uso de dispositivos invasivos e o manejo de feridas (Percival et al., 2015).

As Unidades de Terapia Intensiva (UTIs) possuem um grande número de casos desse tipo de infecção, representando mais de $30 \%$ de todas as internações em UTIs. Isso se deve ao perfil dos pacientes internados neste setor, os quais apresentam reposta imunopatológica comprometida, devido à gravidade da doença, as doenças subjacentes e a maior exposição a procedimentos invasivos. Além destes fatores, o uso de antibióticos de amplo espectro contribui para o desenvolvimento de micro-organismos resistente a diversos fármacos (Alp \& Damani, 2015).

Os tipos mais comuns de IH são as pneumonias associadas à ventilação (PAV), as infecções do trato urinário associadas a cateter (ITUAC), as infecções da corrente sanguínea associada a acesso central (ICSAAC), as infecções de feridas cirúrgicas 
(IFC) e as do trato gastrintestinal (Babady, 2016; Al-Tawfiq \& Tambyah, 2014; Percival et al., 2015).

Os micro-organismos que estão frequentemente envolvidos com IH são as bactérias Gram-positivas como Staphylococcus aureus, Staphylococcus epidermidis e Enterococcus sp.; e as Gram negativas são Escherichia coli, Klebsiella pneumoniae, Proteus mirabilis, Pseudomonas aeruginosa e Acinetobacter baumanni; além desses tem-se as leveduras, especialmente espécies de Candida (Percival et al., 2015).

A pneumonia é a segunda causa mais comum de infecção hospitalar, sendo a primeira em pacientes de UTIs, em maior parte associada a ventilação mecânica. De modo que, os micro-organismos mais isolados em aspirados traqueais são Pseudomonas aeruginosa, Acinetobacter baumannii e Staphilococcus aureus, sendo os bacilos Gram-negativos (BGN) responsáveis por mais de 60\% dos casos (Canzi \& Colacite, 2016).

$\mathrm{O}$ aspirado traqueal é usado em todo o mundo para o diagnóstico de pneumonia associada à ventilação mecânica (PAVM), se tratando de um método não invasivo, que apresenta resultados semelhantes àqueles invasivos no que se refere ao desfecho (Ranzani et al., 2016). Trata-se de um método vantajoso por ser facilmente realizado à beira do leito, de baixo custo e relativamente simples (Frota et al., 2014).

Diante da importância do conhecimento do perfil bacteriano de culturas na construção de protocolos norteadores para o tratamento das infecções hospitalares, assim como diminuir a seleção de bactérias multirresistentes, a presente pesquisa avaliou a ocorrência e o perfil bacteriano de aspirados traqueais coletados em pacientes internados na UTI de um Hospital Universitário do Sertão do Vale do São Francisco.

\section{Metodologia}

O estudo foi realizado através da análise de exames de aspirados traqueais de pacientes internados na UTI de um Hospital Universitário, no período de janeiro a dezembro de 2017 e janeiro a dezembro de 2018. A pesquisa trata-se de um estudo observacional, retrospectivo e descritivo com abordagem quantitativa, cujos dados foram coletados por meio de impressos laboratoriais do próprio serviço, conforme Ribeiro et al., (2019).

O Hospital Universitário apresenta um perfil assistencial de hospital geral de média e alta complexidade à comunidade adulta. É considerado referência em traumas, politraumas, ortopedia, neurocirurgia, cirurgia geral e clínica médica.

Os dados dos exames foram tabulados na planilha do Excel®, sendo divididos em amostras positivas e negativas, e realizada análise descritiva com valores absolutos e em percentuais. Nas amostras positivas foram analisadas as variáveis, ocorrência de bactérias e o perfil de sensibilidade e resistência aos antibióticos testados. Os dados foram organizados e apresentados por meio de gráficos e tabelas.

As identificações das bactérias e os antibiogramas foram executados através do sistema automatizado PHOENIX, da BD, utilizando-se os painéis adequados. De acordo com a metodologia do Clinical and Laboratory Standards Institute (2017 e 2018), os resultados foram classificados em sensível (S) e resistente (R).

O trabalho foi aprovado pelo Comitê de Ética e Deontologia em Estudos e Pesquisas (CEDEP) da Universidade Federal do Vale do São Francisco (UNIVASF) através do CAAE: 66493917.0.0000.5196.

\section{Resultados}

Foram analisadas 307 amostras de aspirados traqueais no ano de 2017, sendo 142 (46,3\%) positivas e 165 (53,7\%) foram negativas. No ano de 2018 foram 319 amostras avaliadas, sendo 213 (66,8\%) positivas e 106 (33,2\%) negativas.

Na Figura 1, observa-se que as espécies bacterianas mais frequentes em aspirados traqueais analisados no período de janeiro a dezembro de 2017, foram Acinetobacter baumanii (41\%), Staphylococcus aureus (21\%), Pseudomonas aeruginosa (14\%) e Klebsiella pneumoniae (13\%), no restante das amostras (11\%) estão as espécies bacterinas menos frequentes. No ano 
de 2018 foram mais frequentes, Acinetobacter baumanii (27\%), Staphylococcus aureus (21\%), Pseudomonas aeruginosa (23\%) e Klebsiella pneumoniae (13,5\%), outras bactéria, menos recorrentes somaram $17 \%$ das culturas.

Figura 1. Ocorrência de bactérias isoladas em aspirados traqueais coletados em pacientes internados em um Hospital Universitário no ano de 2017 e 2018. *Staphylococcus coagulase negativa.

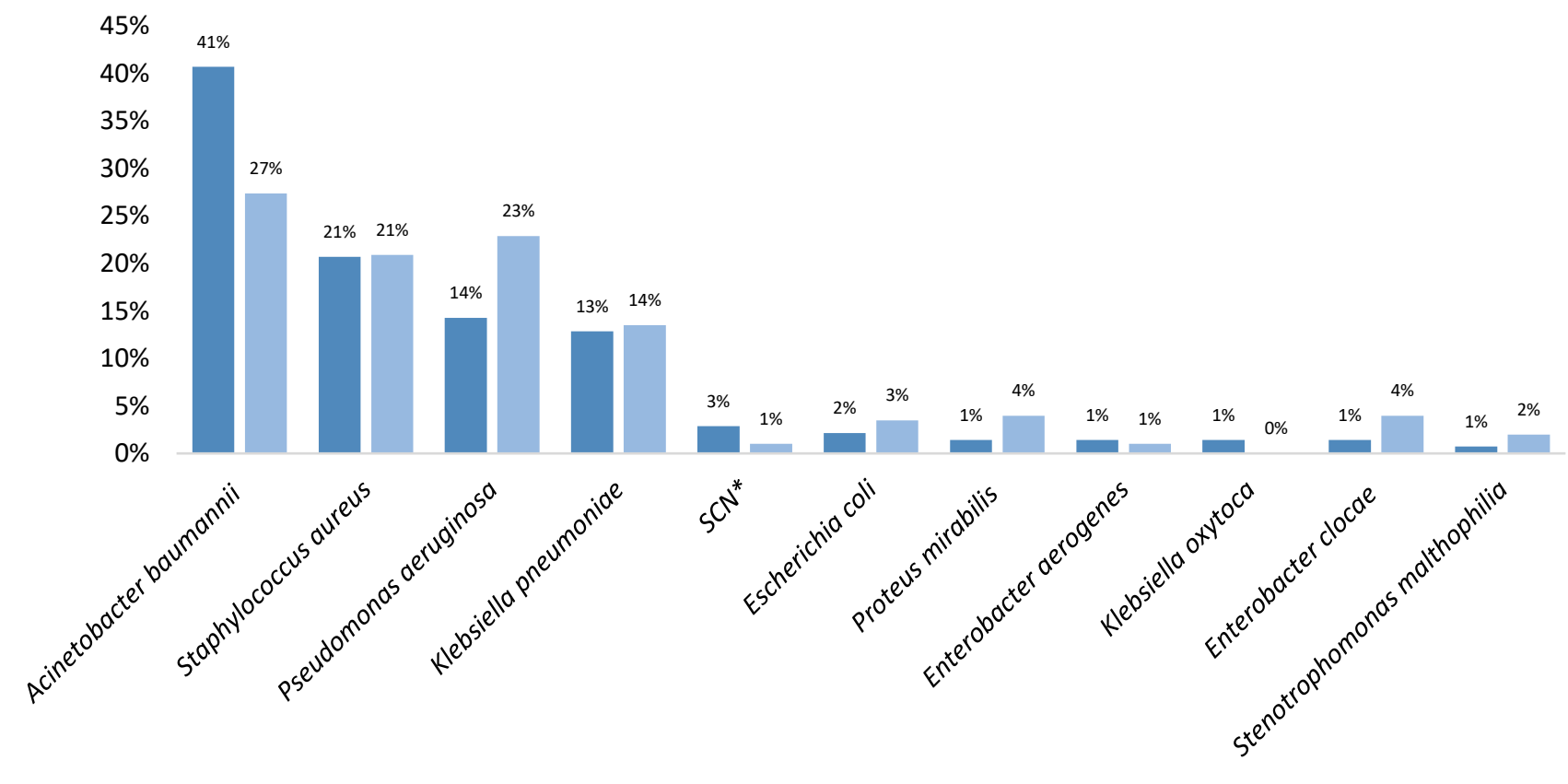

Fonte: Autores

- $2017 \square 2018$

Em relação ao perfil de resistência aos antibióticos, o meropenem e o imipenem, são antibióticos carbapenêmicos utilizados para o tratamento das infecções hospitalares causadas por A. baumannii, o que se observou para ambos foi que em 2017, $92 \%$ dos isolados em aspirados traqueais foram resistentes a essas drogas e que em 2018 essa porcentagem foi de $72 \%$ (Tabela 1). Outra opção é a ampilicina + sulbactam que apresentou 69\% e 51\% de resistência em 2017 e 2018 , respectivamente. A polimixina B também é uma opção, no entanto, não foi testada nos antibiogramas. Outra droga testada foi a colistina e nos dois anos os isolados de A. baumanni foram $100 \%$ sensíveis (Tabela 1).

Para o controle de $S$. aureus podem ser utilizadas a clindamicina, o sulfametoxazol + trimetoprima, oxalicina e vancomicina. A resistência a clindamicina foi de $42 \%$ em 2017 e de $38 \%$ em 2018 (Tabela 1). No ano de 2017, 7\% dos isolados de S. aureus foram resistentes ao sulfametoxazol + trimetoprima e em 2018 foram $2 \%$. Para oxacilina foi observada $45 \%$ e $10 \%$ em 2017 e 2018, respectivamente. Nenhum dos isolados de S. aureus foi resistente à vancomicina nos dois anos observados (Tabela 1).

O meropenem e o imipinem, também podem ser utilizados no tratamento de infecções por P. aeruginosa, para o primeiro antimicrobiano observou-se uma resistência de 20\% em 2017 e 46\% em 2018, o segundo teve $40 \%$ em 2017 e $45 \%$ em 2018 (Tabela 1). A piperacilina + tazobactam é outra combinação utilizada, que em 2017 observou-se não ter nenhum relato de resistência, tendo em 2018, 23\% de isolados resistentes. Para a amicacina, a resistência em 2017 e 2018 foi de $30 \%$ e $33 \%$, respectivamente. Em relação as cefalosporinas, em 2017, P. aerugonosa foi 53\% resistentes a ceftazidima e em 2018 esse número foi de 48\%; para a cefepima, em 2017, a resistência foi de $45 \%$ e de $50 \%$ em 2018. A resistência dessa bactéria ao ciprofloxacino foi de $45 \%$ em 2017 e de $49 \%$ em 2018 e para o levofloxacino em 2017 foi 57\% e em 2018 foi 52\% (Tabela 1). 
Para o tratamento da K. pneumoniae, houve resistência de $27 \%$ em 2017 e $20 \%$ em 2018 ao imipenem. Os isolados resistentes ao meropenem foram $25 \%$ em 2017 e $33 \%$ em 2018. Ao se testar a resistência dos isolados a piperacilina + tazobactam essa foi de $27 \%$ em 2017 e $41 \%$ em 2018. Na avaliação da amicacina não houve isolados resistentes nos dois anos. A polimixina B não foi testada nos isolados. A colistina, nos isolados de K. pneumoniae, dos dois anos apresentou 100\% de sensibilidade entre as amostras (Tabela 1).

Tabela1. Perfil de resistência das espécies bacterianas mais isoladas de aspirados traqueais coletadas de pacientes internados no Hospital Universitário-Univasf, no período de janeiro a dezembro de 2017 e 2018.

\begin{tabular}{|c|c|c|c|c|c|c|c|c|}
\hline \multirow[t]{3}{*}{ Antibióticos } & \multicolumn{8}{|c|}{ Isolados bacterianos } \\
\hline & \multicolumn{2}{|c|}{$\begin{array}{l}\text { Pseudomonas } \\
\text { aeruginosa }\end{array}$} & \multicolumn{2}{|c|}{$\begin{array}{c}\text { Staphylococcus } \\
\text { aureus }\end{array}$} & \multicolumn{2}{|c|}{$\begin{array}{c}\text { Acinetobacter } \\
\text { baumanni }\end{array}$} & \multicolumn{2}{|c|}{$\begin{array}{c}\text { Klebsiella } \\
\text { pneumoniae }\end{array}$} \\
\hline & $2017(\%)$ & $2018(\%)$ & $2017(\%)$ & $2018(\%)$ & $2017(\%)$ & $2018(\%)$ & $2017(\%)$ & $2018(\%)$ \\
\hline Colistina & 0 & 0 & $\mathrm{Nt}$ & $\mathrm{Nt}$ & 0 & 0 & 0 & 0 \\
\hline Meropenem & 20 & 46 & $\mathrm{Nt}$ & $\mathrm{Nt}$ & 93 & 72 & 25 & 33 \\
\hline Imipinem & 40 & 45 & $\mathrm{Nt}$ & $\mathrm{Nt}$ & 93 & 72 & 27 & 20 \\
\hline Ertapenem & $\mathrm{Nt}$ & $\mathrm{Nt}$ & $\mathrm{Nt}$ & $\mathrm{Nt}$ & $\mathrm{Nt}$ & $\mathrm{Nt}$ & 23 & 42 \\
\hline $\begin{array}{l}\text { Piperacilina } \\
\text { tazobactam }\end{array}$ & 0 & 23 & $\mathrm{Nt}$ & $\mathrm{Nt}$ & 96 & 80 & 27 & 41 \\
\hline Ceftazidima & 53 & 48 & $\mathrm{Nt}$ & $\mathrm{Nt}$ & 98 & 77 & 75 & $\mathrm{Nt}$ \\
\hline Cefuroxima & $\mathrm{Nt}$ & $\mathrm{Nt}$ & $\mathrm{Nt}$ & $\mathrm{Nt}$ & $\mathrm{Nt}$ & $\mathrm{Nt}$ & 71 & 100 \\
\hline Cefoxitina & $\mathrm{Nt}$ & $\mathrm{Nt}$ & $\mathrm{Nt}$ & $\mathrm{Nt}$ & $\mathrm{Nt}$ & $\mathrm{Nt}$ & 36 & 28 \\
\hline Ciprofloxacino & 45 & 49 & 54 & 0 & 95 & 80 & 47 & 44 \\
\hline Daptomicina & $\mathrm{Nt}$ & $\mathrm{Nt}$ & $\mathrm{Nt}$ & 0 & $\mathrm{Nt}$ & $\mathrm{Nt}$ & $\mathrm{Nt}$ & $\mathrm{Nt}$ \\
\hline Fosfomicica G6P & $\mathrm{Nt}$ & 100 & $\mathrm{Nt}$ & $\mathrm{Nt}$ & $\mathrm{Nt}$ & $\mathrm{Nt}$ & $\mathrm{Nt}$ & $\mathrm{Nt}$ \\
\hline Amicacina & 30 & 33 & $\mathrm{Nt}$ & $\mathrm{Nt}$ & 31 & 73 & 0 & 0 \\
\hline Gentamicina & 30 & 46 & 4 & 0 & 30 & 56 & 44 & 44 \\
\hline Levofloxacino & 57 & 52 & 0 & $\mathrm{Nt}$ & 95 & 79 & 0 & 42 \\
\hline Aztreonam & 45 & 50 & $\mathrm{Nt}$ & $\mathrm{Nt}$ & 100 & 100 & 33 & 100 \\
\hline Smx+Tmp* & 100 & 100 & 7 & 2 & 90 & 66 & 75 & 67 \\
\hline Cefepima & 45 & 50 & $\mathrm{Nt}$ & $\mathrm{Nt}$ & 96 & 79 & 75 & 63 \\
\hline Amp+Sub** & $\mathrm{Nt}$ & $\mathrm{Nt}$ & $\mathrm{Nt}$ & $\mathrm{Nt}$ & 69 & 51 & 59 & 67 \\
\hline Amox+clav**** & $\mathrm{Nt}$ & $\mathrm{Nt}$ & $\mathrm{Nt}$ & $\mathrm{Nt}$ & $\mathrm{Nt}$ & $\mathrm{Nt}$ & $\mathrm{Nt}$ & 100 \\
\hline Ampicilina & $\mathrm{Nt}$ & $\mathrm{Nt}$ & $\mathrm{Nt}$ & 100 & 100 & 100 & 88 & 100 \\
\hline Azitromicina & $\mathrm{Nt}$ & $\mathrm{Nt}$ & 83 & 100 & 100 & $\mathrm{Nt}$ & $\mathrm{Nt}$ & $\mathrm{Nt}$ \\
\hline Ceftarolina & $\mathrm{Nt}$ & $\mathrm{Nt}$ & $\mathrm{Nt}$ & 0 & $\mathrm{Nt}$ & $\mathrm{Nt}$ & $\mathrm{Nt}$ & $\mathrm{Nt}$ \\
\hline Ceftriaxona & $\mathrm{Nt}$ & $\mathrm{Nt}$ & $\mathrm{Nt}$ & $\mathrm{Nt}$ & 98 & 87 & 75 & 63 \\
\hline Cefotaxima & $\mathrm{Nt}$ & $\mathrm{Nt}$ & $\mathrm{Nt}$ & $\mathrm{Nt}$ & 100 & 100 & 100 & $\mathrm{Nt}$ \\
\hline Cefazolina & $\mathrm{Nt}$ & $\mathrm{Nt}$ & $\mathrm{Nt}$ & $\mathrm{Nt}$ & $\mathrm{Nt}$ & $\mathrm{Nt}$ & $\mathrm{Nt}$ & 57 \\
\hline Clindamicina & $\mathrm{Nt}$ & $\mathrm{Nt}$ & 42 & 38 & $\mathrm{Nt}$ & $\mathrm{Nt}$ & $\mathrm{Nt}$ & $\mathrm{Nt}$ \\
\hline
\end{tabular}




\begin{tabular}{lllcccccc}
\hline Cloranfenicol & $\mathrm{Nt}$ & $\mathrm{Nt}$ & 9 & $\mathrm{Nt}$ & $\mathrm{Nt}$ & 100 & 33 & 0 \\
Eritromicina & $\mathrm{Nt}$ & $\mathrm{Nt}$ & 67 & 43 & $\mathrm{Nt}$ & $\mathrm{Nt}$ & $\mathrm{Nt}$ & $\mathrm{Nt}$ \\
Linezolida & $\mathrm{Nt}$ & $\mathrm{Nt}$ & 0 & 0 & $\mathrm{Nt}$ & $\mathrm{Nt}$ & $\mathrm{Nt}$ & $\mathrm{Nt}$ \\
Minociclina & $\mathrm{Nt}$ & $\mathrm{Nt}$ & $\mathrm{Nt}$ & 0 & 2 & 0 & $\mathrm{Nt}$ & $\mathrm{Nt}$ \\
Oxacilina & $\mathrm{Nt}$ & $\mathrm{Nt}$ & 45 & 10 & 0 & $\mathrm{Nt}$ & $\mathrm{Nt}$ & $\mathrm{Nt}$ \\
Penicilina & $\mathrm{Nt}$ & $\mathrm{Nt}$ & 83 & 100 & $\mathrm{Nt}$ & $\mathrm{Nt}$ & $\mathrm{Nt}$ & $\mathrm{Nt}$ \\
Rifampicina & $\mathrm{Nt}$ & $\mathrm{Nt}$ & 17 & 2 & $\mathrm{Nt}$ & $\mathrm{Nt}$ & $\mathrm{Nt}$ & $\mathrm{Nt}$ \\
Tetracilina & $\mathrm{Nt}$ & $\mathrm{Nt}$ & 6 & 0 & 69 & 42 & 67 & 0 \\
Tigeciclina & $\mathrm{Nt}$ & $\mathrm{Nt}$ & 0 & 0 & 0 & 0 & 8 & 0 \\
Ácido fusídico & $\mathrm{Nt}$ & $\mathrm{Nt}$ & 0 & $\mathrm{Nt}$ & $\mathrm{Nt}$ & $\mathrm{Nt}$ & $\mathrm{Nt}$ & $\mathrm{Nt}$ \\
Moxifloxacino & $\mathrm{Nt}$ & $\mathrm{Nt}$ & 42 & $\mathrm{Nt}$ & $\mathrm{Nt}$ & $\mathrm{Nt}$ & $\mathrm{Nt}$ & $\mathrm{Nt}$ \\
Nitrofurantoina & $\mathrm{Nt}$ & $\mathrm{Nt}$ & 0 & $\mathrm{Nt}$ & $\mathrm{Nt}$ & $\mathrm{Nt}$ & $\mathrm{Nt}$ & $\mathrm{Nt}$ \\
Norfloxacino & $\mathrm{Nt}$ & $\mathrm{Nt}$ & 43 & $\mathrm{Nt}$ & $\mathrm{Nt}$ & $\mathrm{Nt}$ & $\mathrm{Nt}$ & $\mathrm{Nt}$ \\
Teicoplamina & $\mathrm{Nt}$ & $\mathrm{Nt}$ & 0 & $\mathrm{Nt}$ & $\mathrm{Nt}$ & $\mathrm{Nt}$ & $\mathrm{Nt}$ & $\mathrm{Nt}$ \\
Vancomicina & $\mathrm{Nt}$ & $\mathrm{Nt}$ & 0 & 0 & $\mathrm{Nt}$ & $\mathrm{Nt}$ & $\mathrm{Nt}$ & $\mathrm{Nt}$ \\
\hline
\end{tabular}

*Nt: Não testado; Sulmetaxozol + trimetoprima; ** Ampicilina + sulbactam; *** Amoxicilina + clavulanato. Fonte: Autores.

\section{Discussão}

As infecções relacionadas aos cuidados à saúde são as principais causas de morte e incapacidade que pode ser prevenida entre os pacientes hospitalizados (Boev \& Kiss, 2017).

Um estudo que avaliou pacientes admitidos na UTI com tempo de ventilação mecânica invasiva maior que 48h, observou que a $P$. aeruginosa foi o principal micro-organismo isolado (33,7\%), seguida do A. baumannii (22,5\%). Nesse mesmo estudo o $S$. aureus teve uma ocorrência de 6\% e a K. pneumoniaede 5\% (Canzi \& Colacite, 2016). Esses dados são semelhantes àqueles observados no presente trabalho, em que A. baumannii e $P$. aeruginosa apresenteram uma ocorrência elevada. Em outro estudo, realizado na UTI do hospital da Santa Casa de Misericórdia de Goiânia, observou-se para os aspirados traqueais que a taxa mais elevada foi de A. baumannii (37,9\%) seguida de P. aeruginosa (27,6\%) (Mota et al., 2018), corroborando com os dados apresentados no presente trabalho.

S. aureus foi uma das bactérias mais isoladas no presente estudo, $21 \%$ das amostras positivas tanto em 2017 quanto em 2018, corroborando com os resultados de uma pesquisa onde buscou-se identificar a prevalência de cepas de $S$. aureus na UTI de um hospital, nos anos de 2014 e 2015. Nestes anos foram encontradas, em aspirados traqueais, respectivamente $24,5 \%$ e 35,4\% da amosras com S. aureus (Simões et al., 2017).

No Hospital Universitário de Santa Maria, onde foram avaliadas a presença de bactérias produturas de carbapenemase, K. pneumoniae foi a mais isoloda, sendo encontrada principalmente nas secreções traqueais (25\%) (Seibert et al., 2014). Neste trabalho K. pneumoniae teve uma porcentagem de recorrência de 13\% e 13,5\%, em 2017 e 2018, respectivamente.

A resistência a antimicrobianos observadas nesse estudo são explicadas por mecanismos que foram emergindo a partir de uma pressão seletiva e o desenvolvimento de diferentes mecanismos de resistência (Blair et al., 2014; Suárez et al., 2006).

A resistência aos antimicrobianos surge concomitantemente a sua própria descoberta, podendo ser exemplificada com o caso do S. aureus com a introdução das penicilinas em 1940. Entretanto após 2 anos de uso já começaram a ser observadas resistência a essa droga, por meio da produção das chamadas betalactamases. Por volta de 1960, mais de 80\% das cepas de $S$. aureus já eram resistentes a penicilina e como opção de tratamento surge a meticilina, mas tão logo essa droga foi introduzida já se observou resistência ao seu uso (Lowy, 2003). A rápida difusão dessa resistência se dá por no mínimo dois mecanismos: 
disseminação dos clones resistentes e a aquisição do SCCmec (cassete chromosome mec) por uma estirpe anteriormente sensível, o SCCmec codifica os genes mecA ou mecC, responsáveis pela resistência a meticilina e a maioria do antibóticos da classe dos betalactâmicos. O MRSA associado a cuidados de saúde (HA-MRSA) pode levar a bacteremia, pneumonia e menos comumente a infecção de pele e de tecidos moles (Lee et al., 2018).

As betalactamases de espectro extendido (ESBLs), apresentam atividade contra cefotazima, ceftazidima e ceftriaxona. Existe uma grande variedade do gene CTX-M, que codifica as ESBLs, uma delas é a enzima CTX-M15 presente, principalmente, nos isolados de K. pneumoniae de origem nosocomial. Consequentemente ao grande número de bactérias que apresentavam as ESBLs houve um aumento do uso dos antibióticos carbapenêmicos, que por sua vez levou a um aumento de isolados contendo as carbapenemases que inativam uma ampla variedade de betalactamicos incluindo os carbapenêmicos e as cefalosporinas de espectro extendido (Blair et al., 2014).

Em relação a resistência aos carbapenêmicos observada nas amostras de Acinetobacter baumannii, Pseudomonas aeruginosa e K. pneumoniae, acredita-se que os principais mecanismos associados são a produção de uma betalactamase e a diminuição de porinas que leva a diminuição da permeabilidade da membrana externa. As betalactamases envolvidas nesse processo são as AmpC e carbapenemases, a primeira é responsável pela resistência das cefalosporinas de terceira geração, ao aztreoanam e aos inibidores da betalactamase; e as carbamenemases podem ser subdivididas em dois tipos: serina-betalactamases e metalo-betalactamases. Dentre as serina-betalactamases destaca-se as do tipo KPC que apresentam atividade hidrolítica contra aminopenicilinas, ureidopenicilinas (piperacilina), aztreonam, carbapenêmicos e as cefalosporinas de terceira geração. A respeito das metalo-betalactamases, mais comumente encontradas no A. baumanni e na P. aeruginosa, e geram resistência aos betalactâmicos, aminoglicosídeos e quinolonas (Suárez et al., 2006).

A resistência as Quinolonas observada nos principais isolados do presente estudo devem-se as mutações nos alvos das drogas que são as enzimas DNA girase e a DNA topoisomerase IV; além dessa, existem mutações que reduzem o acúmulo da droga e plasmidios que protegem as células dos seus efeitos, alterações na permeabilidade da membrana e mecanismos de efluxo (Jacoby, 2005).

\section{Conclusão}

As infecções hospitalares desencadeadas pelas bactérias e os seus mecanismos de resistência evoluem paralelamente ao uso dos antibióticos e são objetos de grande preocupação para a comunidade científica e para a população em geral, uma vez que a elevada ocorrência dessas infecções e a dificuldade em trata-las geram um enorme prejuízo social e econômico. Dessa forma, estudos como este, que apresentam a ocorrência bacteriana e o perfil de resistência dessas bactérias contribuem para o melhor entendimento da situação, consequentemente auxiliam na elaboração de condutas terapêuticas racionais, e principalmente, fomenta a iniciativa de que sejam elaborados meios de prevenção. Estudos futuros complementares serão realizados de forma direcionada a infecções associadas a ventilação mecânica e contribuirão com o Setor de Controle de Infecções Hospitalares para a construção de protocolos empíricos de antibiótico direcionados a Unidade de Terapia Intensiva.

\section{Referências}

Al-Tawfiq, J. A., \& Tambyah, P. A. (2014). Healthcare associated infections (HAI) perspectives. Journal of Infection and Public Health, 7(4), 339-344. https://doi.org/10.1016/j.jiph.2014.04.003

Alp, E., \& Damani, N. (2015). Healthcare-associated infections in Intensive Care Units: epidemiology and infection control in low-to-middle income countries. The Journal of Infection in Developing Countries, 9(10), 1040-1045. https://doi.org/10.3855/jidc.6832

Babady, N. E. (2016). Hospital-Associated Infections. Microbiology Spectrum, 4(3). https://doi.org/10.1128/microbiolspec.dmih2-0003-2015

Blair, J. M. A., Webber, M. A., Baylay, A. J., Ogbolu, D. O., \& Piddock, L. J. V. (2014). Molecular mechanisms of antibiotic resistance. Nature Reviews Microbiology, 13(1), 42-51. https://doi.org/10.1038/nrmicro3380 
Research, Society and Development, v. 10, n. 14, e23101421550, 2021

(CC BY 4.0) | ISSN 2525-3409 | DOI: http://dx.doi.org/10.33448/rsd-v10i14.21550

Boev, C., \& Kiss, E. (2017). Hospital-Acquired Infections. Critical Care Nursing Clinics of North America, 29(1), 51-65. https://doi.org/10.1016/j.cnc.2016.09.012

Calik Basaran, N., \& Ascioglu, S. (2017). Epidemiology and management of healthcare-associated bloodstream infections in non-neutropenic immunosuppressed patients: a review of the literature. Therapeutic Advances in Infectious Disease, 4(6), 171-191. https://doi.org/10.1177/2049936117733394

Canzi, K. R., \& Colacite, J. (2016). Frequência de pneumonia associada à ventilação mecânica com base em resultados de culturas quantitativas de secreções traqueais [Review of Frequência de pneumonia associada à ventilação mecânica com base em resultados de culturas quantitativas de secreções traqueais]. RBAC, 48(2), 118-122. http://sbac.org.br/rbac/wp-content/uploads/2016/06/ARTIGO-3_RBAC-48-2-2016-ref.-386.pdf

Cardoso, T., Almeida, M., Friedman, N. D., Aragão, I., Costa-Pereira, A., Sarmento, A. E., \& Azevedo, L. (2014). Classification of healthcare-associated infection: a systematic review 10 years after the first proposal. BMC Medicine, 12(1). https://doi.org/10.1186/1741-7015-12-40

Frota, O. P., Ferreira, A. M., Barcelos, L. da S., Watanabe, E., Carvalho, N. C. P., Rigotti, M. A., Frota, O. P., Ferreira, A. M., Barcelos, L. da S., Watanabe, E., Carvalho, N. C. P., \& Rigotti, M. A. (2014). Collection of tracheal aspirate: safety and microbiological concordance between two techniques. Revista Da Escola de Enfermagem Da USP, 48(4), 618-624. https://doi.org/10.1590/S0080-623420140000400007

Jacoby, G. A. (2005). Mechanisms of resistance to quinolones. Clinical Infectious Diseases: An Official Publication of the Infectious Diseases Society of America, 41 Suppl 2, S120-6. https://doi.org/10.1086/428052

Lee, A. S., de Lencastre, H., Garau, J., Kluytmans, J., Malhotra-Kumar, S., Peschel, A., \& Harbarth, S. (2018). Methicillin-resistant Staphylococcus aureus. Nature Reviews. Disease Primers, 4(18033), 18033. https://doi.org/10.1038/nrdp.2018.33

Lowy, F. D. (2003). Antimicrobial resistance: the example of Staphylococcus aureus. Journal of Clinical Investigation, 111(9), 1265-1273. https://doi.org/10.1172/jci18535

Mota, F. S. da, Oliveira, H. A. de, \& Souto, R. C. F. (2018). Profile and prevalence of antimicrobial resistance of negative-Gram bacteria isolated from intensive care patients. Revista Brasileira de Análises Clínicas, 50(3). https://doi.org/10.21877/2448-3877.201800740

Percival, S. L., Suleman, L., Vuotto, C., \& Donelli, G. (2015). Healthcare-associated infections, medical devices and biofilms: risk, tolerance and control. Journal of Medical Microbiology, 64(Pt_4), 323-334. https://doi.org/10.1099/jmm.0.000032

Ranzani, O. T., Forte, D. N., Forte, A. C., Mimica, I., \& Forte, W. C. N. (2016). The value of antibody-coated bacteria in tracheal aspirates for the diagnosis of ventilator-associated pneumonia: a case-control study. Jornal Brasileiro de Pneumologia, 42(3), 203-210. https://doi.org/10.1590/s1806-37562015000000244

Ribeiro, T. de S., Ribeiro, R. A. A. dos S., Batista, K. S., Ricarte de Aquino, S., \& Naue, C. R. (2019). Ocorrência e perfil bacteriano de culturas coletadas em pacientes internados na unidade de terapia intensiva em um hospital terciário. HU Revista, 45(2), 122-133. https://doi.org/10.34019/1982-8047.2019.v45.25933

Seibert, G., Hörner, R., Meneghetti, B., Alves, R., Frasson, N., Forno, D., \& Salla, A. (2014). Einstein. 12(3), 282-288. https://doi.org/10.1590/S167945082014AO3131

Simões, A. C. A., Miranda, M. M., \& Teixeira, C. D. (2017). Resistência a antimicrobianos em CEPAS DE Staphylococcus aureus isoladas da UTI de um hospital achde Coeiro de Itapemirim - ES. Revista Univap, 22(40), 363. https://doi.org/10.18066/revistaunivap.v22i40.864

Suarez, C. J., Kattan, J. N., Guzman, A. M., \& Villegas, M. V. (2006). Resistance mechanisms to carabapenems in P. aeuriginosa, Acinetobacter baumannii and Enterobacteriaceae and strategies for prevention and control. Infectio, 10(2), 85-93. Scielo. http://www.scielo.org.co/scielo.php?pid=S0123$93922006000200006 \&$ script=sci_abstract\&tlng=en

World Health Organization. (2016). Guidelines on core components of infection prevention and control programmes at the national and acute health care facility level. World Health Organization. 